\title{
The Bidirectional Associations Between Insomnia and Psychotic-Like Experiences Before and During the COVID-I 9 Pandemic
}

\author{
Dongfang Wang ${ }^{1,2, *}$ \\ Liang Zhou (D) ${ }^{1, *}$ \\ Jiali Wang ${ }^{3}$ \\ Meng Sun $\mathbb{D}^{\prime}$
}

'Department of Social Psychiatry, the Affiliated Brain Hospital of Guangzhou Medical University (Guangzhou Huiai Hospital), Guangzhou, People's Republic of China; ${ }^{2}$ School of Psychology, Centre for Studies of Psychological Applications, Guangdong Key Laboratory of Mental Health and Cognitive Science, Ministry of Education Key Laboratory of Brain Cognition and Educational Science, South China Normal University, Guangzhou, People's Republic of China; ${ }^{3}$ Xiangya School of Public Health, Central South University, Changsha, People's Republic of China

*These authors contributed equally to this work
Correspondence: Meng Sun Department of Social Psychiatry, the Affiliated Brain Hospital of Guangzhou Medical University (Guangzhou Huiai Hospital), 36 Mingxin Road, Fangcun, Liwan District, Guangzhou, Guangdong, 510370, People's Republic of China Email sunmengjw070I@I63.com
Study Objectives: Previous literature supports that insomnia is predictive of subsequent psychotic-like experiences (PLEs) in the general population. However, there is a lack of empirical data on the reverse causality between the two variables and on the correlation between the symptom severity of insomnia and PLEs. This study aimed to explore the bidirectional associations between insomnia and PLEs before and during the COVID-19 pandemic.

Methods: A total of 938 students aged 14-25 years completed both waves of the survey before and during the pandemic (the first wave: October 2019 to November 2019; the second wave: April 2020 to May 2020). PLEs were assessed using the 15-item positive subscale of the community assessment of psychic experiences (CAPE-P15), and insomnia was assessed using three questions on difficulty initiating sleep, difficulty maintaining sleep, and early morning awakening.

Results: Students with baseline insomnia were more likely to exhibit new-onset PLEs during the pandemic (OR: 5.13, 95\% CI: 2.54-10.38), while no significant predictive effect of insomnia was found for the persistence and severity of PLEs. Meanwhile, baseline PLEs not only predicted the presence of insomnia during the pandemic (OR: 2.14, 95\% CI: 1.25-3.65) but also correlated with its severity (B: $0.89,95 \%$ CI: $0.47-1.31$ ).

Conclusion: The study provides the first piece of evidence for the bidirectional association between insomnia and PLEs in the general population. However, although insomnia has an important predictive role in the occurrence of PLEs, it does not predict the persistence and development of PLEs, suggesting that there is a more complex mechanism underlying the process. Keywords: insomnia, psychotic-like experiences, COVID-19

\section{Introduction}

A great volume of literature has robustly supported the co-occurrence of insomnia and psychosis, including in clinical populations, those at ultra-high-risk for psychosis, and the general population. ${ }^{1-4}$ While research in clinical populations (patients with schizophrenia or other psychotic disorders) aims to explore the interaction between insomnia and psychosis in predicting clinical symptomatology, functions and treatment, ${ }^{5,6}$ studies in non-clinical populations are essential to understanding their potential relationship in the absence of antipsychotic medication and institutionalisation effects, ${ }^{7}$ which also provides information on prevention at an early stage.

As part of the continuum of psychosis, psychotic-like experiences (PLEs) exist to varying degrees in the general population ${ }^{8,9}$ and are greatly associated with subsequent full-blown psychotic disorders. ${ }^{10,11}$ So far, the association between 
insomnia and PLEs has been heavily supported by epidemiological surveys, ${ }^{1}$ most of which used a cross-sectional study design and thus did not provide evidence for causality. Only a small amount of longitudinal evidence suggests that there is a predictive effect of insomnia on future PLEs, with a small-to-medium effect size. ${ }^{12-14}$ However, some limitations in these studies obscure further clarification of this relationship. One study involved a small proportion of those potentially eligible $(\mathrm{N}=106)$, suggesting sampling bias, which weakened its conclusion. ${ }^{12}$ The other two studies used data from the same group of subjects from the 2000 British National Psychiatric Morbidity Survey. ${ }^{13,14}$ They evaluated PLEs with a small number of items (one item for paranoia, and two items for hallucination) and did not explore the dose-dependent relationship between insomnia and PLEs, with binary variables used to assess symptoms. Moreover, none of the above studies explored whether temporally primary PLEs are associated with an increased risk of subsequent insomnia. $^{1,2}$

According to previous research, the relationship between insomnia and PLEs is mediated by changes in negative affect, such as anxiety, worry and depression. ${ }^{13,15}$ At the end of December 2019, a novel coronavirus disease 2019 (COVID-19) broke out in the Chinese city of Wuhan and was later declared a public health emergency of international concern by the World Health Organization on January 30, 2020. ${ }^{16}$ Extreme stressors due to the pandemic are reported to have aggravated a series of negative affects, ${ }^{17,18}$ as well as insomnia ${ }^{19}$ and PLEs, ${ }^{19}$ suggesting that the pathological process underlying insomnia and PLEs may have been accelerated. However, there is still a lack of research on the changes in insomnia and PLEs during the pandemic, as well as on the relationship between insomnia and PLEs under the impact of COVID-19.

In this study, we explored the changes in prevalence of insomnia and PLEs before and during the pandemic. We also investigated different changing patterns of insomnia and PLEs, and examined bidirectional associations between PLEs and insomnia before and during the pandemic.

\section{Methods}

\section{Participants and Procedure}

Our data were collected from a two-wave web-based longitudinal survey among adolescents before and during the COVID-19 pandemic. From October 2019 to November 2019, a convenience sample of students was recruited from five technical secondary schools and six colleges in four provinces (Guangdong, Henan, Hunan, Zhejiang) in China. From April 2020 to May 2020, the second wave of the survey was conducted in the same population. All participants were full-time students enrolled in the technical schools or colleges and completed both waves of the survey by scanning the Quick Response (QR) code of the questionnaire with mobile phones. Participants completed the baseline survey at school during their psychological health course and registered their contact information if they were willing to participate in the subsequent survey. The second wave of the survey was conducted by sending QR codes to those who had registered their contact information at the baseline survey, as all students were quarantined at home during the pandemic. Other details of the procedures are described in our previous study. ${ }^{20}$

All students (or their guardians, if necessary) filled out the electronic informed consent form before starting each webbased survey. This study was carried out in accordance with the Helsinki Declaration as revised in 1989 and approved by the Human Research Ethics Committee of the Affiliated Brain Hospital of Guangzhou Medical University.

\section{Measures}

\section{Socio-Demographic Characteristics}

Socio-demographic data collected for the survey included sex, age, ethnicity, family income, parental marital status,"left-behind" child status (referring to those left behind in their hometown by one or both of their migrant worker parents for more than half a year), ${ }^{21}$ single child status, history of mental disorders, physical conditions (having at least one of the following: arthritis, angina, asthma, diabetes, visual impairment or hearing problems), ${ }^{22}$ and family history of mental disorders. Pandemic-related information, collected at the second wave of the study, included residency location (urban, town, or rural) during the COVID-19 pandemic, whether or not the participant had been infected with COVID-19, and whether or not the participant had relatives or acquaintances (eg, friends, neighbors, classmates, or family members) infected with COVID-19.

\section{Insomnia}

Three items were used to measure symptoms related to insomnia: difficulty initiating sleep, difficulty maintaining sleep, and early morning awakening over the past month. Response to each item were recorded on a five-point Likert scale, ranging from 1 - rarely or never, 2 - mild, 
3 - moderate, 4 - severe, to 5 - very severe. Higher total scores indicated more severe insomnia symptoms. Consistent with previous studies, ${ }^{23}$ participants who scored 4 (severe) or 5 (very severe) on any item were considered to have probable clinical insomnia. Cronbach's $\alpha$ of these items in two waves of the survey were 0.69 and 0.72 , respectively.

\section{PLEs}

The 15-item positive subscale of the community assessment of psychic experiences (CAPE-P15) was used to assess PLEs over the preceding month. ${ }^{24}$ All 15 items cluster into three dimensions: persecutory ideation (PI), bizarre experiences (BEs), and perceptual abnormalities (PAs). The response to each item ranges from 1 - never, 2 - sometimes, 3 - often, to 4 -nearly always. The weighted score was calculated by dividing the total score by the number of items completed. The Chinese version of the CAPE-P15 has showed satisfactory psychometric

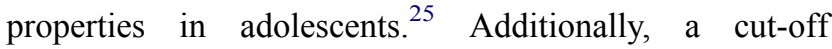
weighted score of 1.57, which was identified in our previous research, ${ }^{26}$ was used for detecting genuine PLEs in this study. In this sample, Cronbach's $\alpha$ for the total score was 0.90 before the pandemic and 0.93 after the pandemic.

\section{Statistical Analyses}

Participants who completed both waves of the survey were included in analysis. To ensure the quality of the survey responses, those with response times of less than 5 minutes (in either wave of the survey) were excluded. All analyses were conducted using SPSS 23.0. ${ }^{27}$ A two-sided $p<0.05$ was considered statistically significant.

First, socio-demographic characteristics and the prevalence of PLEs and insomnia were analyzed. McNemar's test was then used to compare the prevalence of insomnia and PLEs before and during the pandemic. As previous research has suggested that the pandemic had a greater psychological impact on adolescents living in Hubei Province and those having relatives or acquaintances infected with COVID-19, ${ }^{17,18,20,28}$ McNemar's test was also conducted among these subgroups and among the remaining participants after excluding these subgroups.

Second, chi-square tests were used to compare the prevalence of PLEs during the pandemic between participants with insomnia at baseline and those without, as well as the prevalence of insomnia during the pandemic between participants with PLEs at baseline and those without. Intercept models were established initially to evaluate the school/college-level heterogeneity for the presence of PLEs and insomnia, with no significant heterogeneity found ( $p=0.756$ and 0.732 ). The presence of PLEs and insomnia during the pandemic was then analyzed using generalized linear models (GLMs), with logit as a link function. The unadjusted models were initially fitted using maximum likelihood estimation, and included fixed effects for baseline presence of insomnia, baseline presence of PLEs, and baseline presence of insomnia by baseline presence of PLEs interaction. All sociodemographic variables (sex, age, ethnicity, family income, parental marital status, "left-behind" child status, single child status, history of mental disorders, physical conditions, family history of mental disorders, and residence location) were then entered as control variables in the adjusted models. The odds ratio (OR) and 95\% confidence interval (CI) were calculated to quantify the strength of the association.

Finally, we investigated the predictive value of baseline severity of insomnia/PLEs for the severity of PLEs/insomnia during the pandemic. Spearman correlation analysis was initially used to examine the correlation between CAPE-P15 total scores and insomnia total scores across the two waves of the survey. The GLMs were then used for analysis, with the CAPE-P15 total scores and insomnia total scores during the pandemic as the dependent variables. The unadjusted models included fixed effects for baseline CAPE-P15 total scores, baseline insomnia total scores, and baseline CAPE-P15 total scores by baseline insomnia total scores interaction. All sociodemographic variables were also included as control variables in the adjusted models. GLMs were further fitted to explore the bidirectional associations between three dimensions of the CAPE-P15 (PI, BEs, PAs) and insomnia with a consistent approach. Unstandardized coefficients (B) and 95\% confidence intervals for $\mathrm{B}(95 \% \mathrm{CI})$ were calculated.

\section{Results}

\section{Sample Characteristics}

Two thousand two hundred and sixty-five students completed the baseline survey, with $96.5 \%$ of them registering their contact information. Of these participants, a total of 938 participants (response rate $=41.9 \%$ ) completed both waves of the survey with response times greater than 5 minutes for each wave. Details of the data collection are described in our previous paper. ${ }^{27}$ No significant differences were found in the prevalence of insomnia (34.0\% vs $\left.34.7 \%, \chi^{2}=0.13, p=0.763\right)$ or PLEs $(16.0 \%$ vs $18.2 \%$, 
$\left.\chi^{2}=1.81, p=0.194\right)$ between students who followed up and those lost to follow-up.

All of the students were from 14 to 25 years old, and over half of them were female. Twenty students lived in Hubei province, China, which was assessed as a high-risk area during the COVID-19 pandemic by the World Health Organization. ${ }^{27}$ No students reported having been infected with COVID-19, and eight participants reported that they had relatives or friends infected with COVID-19. Other demographic characteristics are described in our previous paper. $^{20}$

\section{Changes of Insomnia and PLEs During the COVID-I9 Pandemic}

There was no significant difference in the prevalence of insomnia between the two waves of the survey $(9.9 \%$ vs $8.6 \%, \chi^{2}=1.04, p=0.307$ ), while the prevalence of PLEs was significantly lower during the pandemic than before the pandemic $\left(16.0 \%\right.$ vs $\left.5.0 \%, \chi^{2}=72.76, p<0.001\right)$. The results remained similar after excluding participants living in Hubei province during the pandemic or who had relatives or friends infected with COVID-19 (insomnia: $9.9 \%$ vs $8.6 \%, \chi^{2}=1.06$, $p=0.303$; PLEs: $15.8 \%$ vs $4.7 \%, \chi^{2}=71.94, p<0.001$ ).

McNemar's test was limited by the small size of the two subgroups (participants living in Hubei province during the pandemic or with relatives or friends infected with COVID19). However, those living in Hubei province during the pandemic showed a numerically higher prevalence of insomnia $(15.0 \%$ vs $10.0 \%)$ and lower prevalence of PLEs $(15.0 \%$ vs $25.0 \%$ ) during the pandemic. Of those with relatives or friends infected with COVID-19, only one student reported PLEs and one reported insomnia before the pandemic, and one student reported PLEs and no students reported insomnia during the pandemic. Considering the much higher pressure these participants suffered, they do not represent the majority of adolescents in China during the COVID-19 pandemic and could have skewed the overall data. The small number of these individuals also limited further analysis. Hence, we excluded them for the subsequent analyses. Sociodemographic characteristics for the remaining participants are presented in Table 1.

\section{Prediction for the Presence of PLEs and Insomnia}

In this sample, students with insomnia at baseline reported a higher prevalence of PLEs during the pandemic (13.3\% vs $\left.3.8 \%, \chi^{2}=16.44, p<0.001\right)$. Those with PLEs at
Table I Demographic Characteristics of the Participants $(\mathrm{N}=$ 910)

\begin{tabular}{|c|c|c|c|}
\hline Characteristics & & $\mathbf{N}$ & $\%$ \\
\hline \multirow[t]{2}{*}{ Sex } & Female & 633 & 69.6 \\
\hline & Male & 277 & 30.4 \\
\hline Age, year & Mean (SD) & $17.66(1.56)$ & \\
\hline \multirow[t]{2}{*}{ Ethnicity } & $\operatorname{Han}^{\mathrm{a}}$ & 817 & 89.8 \\
\hline & Others & 93 & 10.2 \\
\hline \multirow[t]{3}{*}{ Residence location } & Urban & 201 & 22.1 \\
\hline & Town & 250 & 27.5 \\
\hline & Rural & 459 & 50.4 \\
\hline \multirow{5}{*}{$\begin{array}{l}\text { Family income (RMB per } \\
\text { month) }\end{array}$} & $<1000$ & 36 & 4.0 \\
\hline & $1000-3000$ & 288 & 31.6 \\
\hline & $3000-5000$ & 311 & 34.2 \\
\hline & $5000-10,000$ & 219 & 24.1 \\
\hline & $>10,000$ & 56 & 6.2 \\
\hline \multirow[t]{2}{*}{ Parental marital status } & Married & 800 & 87.9 \\
\hline & $\begin{array}{l}\text { Not currently } \\
\text { married }^{\mathrm{b}}\end{array}$ & 110 & 12.1 \\
\hline "Left-behind" child status & Yes & 438 & 48.1 \\
\hline Single child status & Yes & 177 & 19.5 \\
\hline $\begin{array}{l}\text { History of mental } \\
\text { disorders }\end{array}$ & Yes & 10 & I.I \\
\hline Chronic physical illness $^{c}$ & Yes & 101 & II.I \\
\hline $\begin{array}{l}\text { Family history of mental } \\
\text { disorders }\end{array}$ & Yes & 39 & 4.3 \\
\hline
\end{tabular}

Notes: ${ }^{\mathrm{a}} \mathrm{Han}$ is the major ethnic group in China. ${ }^{\mathrm{b}}$ Not currently married included separated, divorced, and widowed. 'Chronic physical conditions referred to having at least one of the following conditions: arthritis, angina, asthma, diabetes, visual impairment, or hearing problems.

baseline also reported a higher prevalence of insomnia during the pandemic $\left(20.8 \%\right.$ vs $6.3 \%, \chi^{2}=32.82, p<$ 0.001). Thirty-seven students (4.1\%) reported both PLEs and insomnia at baseline.

The changing patterns of insomnia and PLEs before and during the pandemic are presented in Figure 1. As shown in Table 2, after adjusting for all demographic characteristics, the presence of insomnia at baseline was still significantly associated with the presence of PLEs during the pandemic $(\mathrm{OR}=5.13,95 \% \mathrm{CI}=2.54-10.38)$, with a significant interaction effect between baseline insomnia and baseline PLEs (OR $=0.22,95 \% \mathrm{CI}=$ 0.08-0.57). Baseline presence of PLEs was also significantly associated with the presence of insomnia during the pandemic $(\mathrm{OR}=2.14,95 \% \mathrm{CI}=1.25-3.65)$, with no 
A

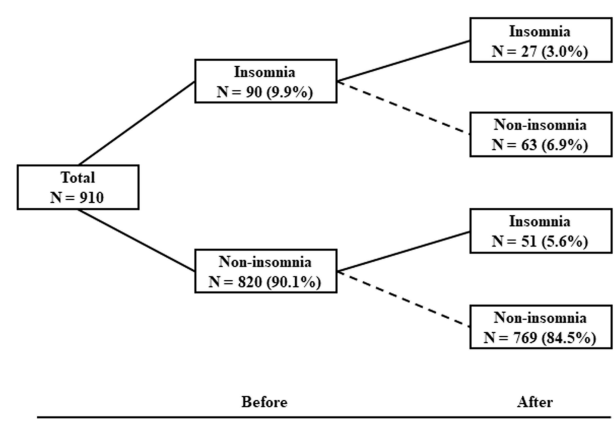

B

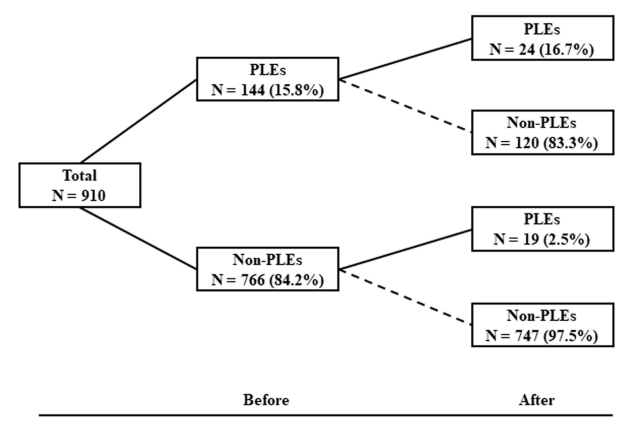

Figure I Changing patterns of PLEs and insomnia before and during the COVID-I9 pandemic. (A) Changing patterns of insomnia. (B) Changing patterns of PLEs. Abbreviation: PLEs, psychotic-like experiences.

interaction effect found $(p=0.063)$. Figure 2 exhibits the moderating effect of baseline PLEs in the relationship between baseline insomnia and PLEs during the pandemic. For students without PLEs at baseline, baseline insomnia played a significant predictive role in PLEs during the pandemic $(\mathrm{OR}=5.13,95 \% \mathrm{CI}=2.54-10.38)$, while no significant association was found between baseline insomnia and PLEs during the pandemic among those with PLEs at baseline $(p=0.737)$.

\section{Prediction for the Severity of PLEs and Insomnia}

Mild-to-moderate correlations were found between insomnia total scores and CAPE-P15 total scores across the two waves of the survey $(r=0.26-0.37$, all $p<0.001)$, between baseline CAPE-P15 total scores and the second CAPE-P15 total scores $(r=0.41, p<0.001)$, and between baseline insomnia total scores and the second insomnia total scores $(r=0.47, p<0.001)$ (see Supplemental Material Table A1). As shown in Table 3, the baseline insomnia total scores failed to predict the CAPE-P15 total scores during the pandemic ( $p=0.397)$, while the baseline CAPE-P15 total scores remained significantly correlated to the insomnia total scores during the pandemic $(\mathrm{B}=0.89,95 \% \mathrm{CI}=0.47-1.31)$ even after adjusting for demographic characteristics.

The bidirectional associations between three dimensions of the CAPE-P15 (PI, BEs, PAs) and insomnia were further explored (see Supplemental Material_Table A2-A4). After adjusting for demographic characteristics, all baseline insomnia total scores were not significantly associated with the second scores of any dimension on the CAPE-P15 during the pandemic, while baseline scores of all PI, BEs, and PAs showed significantly predictive effects on the second insomnia total scores during the pandemic $(\mathrm{B}=0.48-1.00$, all $p<0.01)$. An interaction effect was also found between baseline PAs scores and baseline insomnia total scores in predicting PAs scores during the pandemic.

\section{Discussion}

In this study, we explored the changes in insomnia and PLEs before and during the COVID-19 pandemic in a sample of adolescents, with four different developmental trajectories of insomnia and PLEs identified. We also examined the bidirectional associations between insomnia and PLEs using a prospective longitudinal study design.

Table 2 Prediction for the Presence of PLEs and Insomnia During the COVID-I9 Pandemic $(N=910)$

\begin{tabular}{|l|l|l|l|l|}
\hline \multirow{2}{*}{} & \multicolumn{2}{|l|}{ PLEs During the Pandemic } & \multicolumn{2}{l|}{ Insomnia During the Pandemic } \\
\cline { 2 - 6 } & Crude OR (95\% CI) & Adjust OR (95\% CI) & Crude OR (95\% CI) & Adjust OR (95\% CI) \\
\hline Baseline insomnia & $5.20(1.80,15.04)$ & $5.13(2.54,10.38)$ & $3.53(1.61,7.76)$ & $3.66(1.97,6.81)$ \\
Baseline PLEs & $9.43(4.50,19.79)$ & $10.43(6.39,17.05)$ & $2.18(1.10,4.31)$ & $2.14(1.25,3.65)$ \\
Baseline insomnia $\times$ Baseline PLEs & $0.24(0.06,1.00)$ & $0.22(0.08,0.57)$ & $2.12(0.65,6.91)$ & $2.43(0.95,6.19)$ \\
\hline
\end{tabular}

Notes: adjusting for demographic characteristics (sex, age, ethnicity, residence location, family income, parental marital status, "left-behind" child status, single child status, history of mental disorders, chronic physical illness, family history of mental disorders), and a two-sided $\mathrm{p}<0.05$ was considered statistically significant. Abbreviation: PLEs, psychotic-like experiences. 


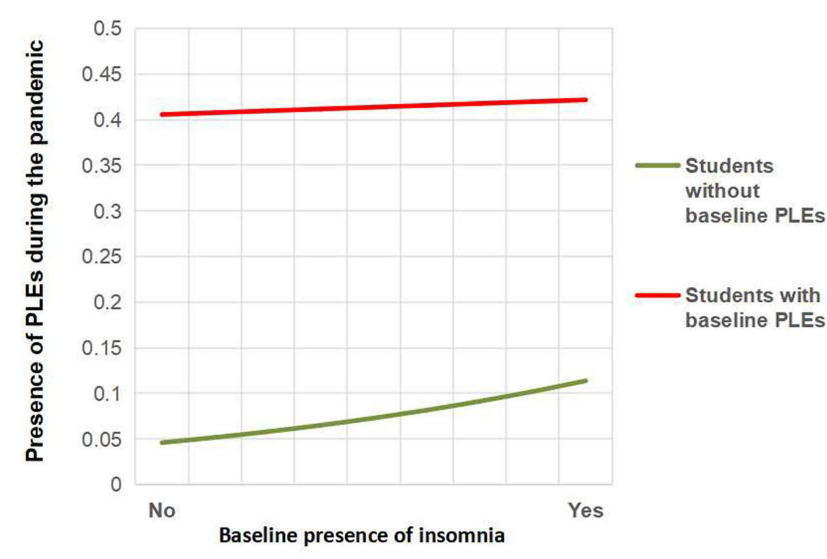

Figure 2 Interaction effect of baseline PLEs and baseline insomnia in predicting PLEs during the COVID-19 pandemic.

Abbreviation: PLEs, psychotic-like experiences.

To our knowledge, this is the first study on the reciprocal predictive relationship between insomnia and PLEs in the general population.

In this sample, the prevalence of insomnia during the pandemic did not change significantly compared to before the pandemic. While the insomnia prevalence before the pandemic is consistent with previous data $(6-10 \%)$ from the DSM-5, ${ }^{29}$ the prevalence during the pandemic seems much lower than that found in a recent meta-analysis $(23.87 \%) .{ }^{30}$ In another recent meta-analysis, the subgroup analysis found that reports from China $(\mathrm{K}=18, \mathrm{n}=$ $28,000)$ yielded a even higher prevalence of sleep problems (26.5\%) during the COVID-19 pandemic. $^{31}$ However, there is high heterogeneity among these original studies. While a research from Italy has reported a similar prevalence $(7.3 \%)$ of insomnia, ${ }^{32}$ the prevalence in a Chinese sample is more than twice as high as our results $(18.2 \%$ vs $8.6 \%) .{ }^{33}$ Different sampling times may partly explain the difference. The previous study assessed insomnia prevalence in February, at the peak of the pandemic in
China, while we conducted the second wave of the survey from April to May 2020. The high attrition rate may also have had an impact on the prevalence in our study, for those with severe mental problems were more likely to be lost to follow-up. According to the psychosis pronenesspersistence-impairment model, ${ }^{34}$ most PLEs are transitory and disappear over time, and only a small portion persists and develops into clinical psychosis. Hence, a significantly decreased prevalence of PLEs may not adequately reflect the impact of the COVID-19 pandemic. However, the incidence within 6 months during the pandemic (0.021) was a slightly higher than the annual incidence reported in previous studies $(0.015-0.020)$ in the same age group, suggesting the probable actual impact from the COVID19 pandemic.

Regarding the prediction of PLEs, the presence of insomnia before the pandemic was found to be associated with about fivefold higher risk of new-onset PLEs during the COVID-19 pandemic. Evidence from previous research also supports this predictive relationship, ${ }^{13,14}$ albeit with a much weaker correlation (OR $=1.17-1.65)$. This may be due to the much longer time intervals (18 months) and the limited types of PLEs assessed in previous research. Additionally, the COVID-19 pandemic may also have strengthened the relationship through elevated levels of depression and anxiety, which have been found to be mediators in this relationship. ${ }^{2}$ However, the presence of insomnia before the pandemic failed to predict the persistence of PLEs during the pandemic among participants with baseline PLEs. Although Freeman et $\mathrm{al}^{13}$ found that baseline insomnia was predictive of the persistence of paranoia in their study, the effect size also appeared very small $(r=0.05)$. All the above results point toward the following conclusion: although PLEs may share a common pathogenesis with insomnia, the

Table 3 Prediction for the Severity of PLEs and Insomnia $(N=910)$

\begin{tabular}{|c|c|c|c|c|}
\hline & \multicolumn{2}{|c|}{$\begin{array}{l}\text { CAPE-PI } 5 \text { Total Scores During } \\
\text { the Pandemic }\end{array}$} & \multicolumn{2}{|c|}{$\begin{array}{l}\text { Insomnia Total Scores During the } \\
\text { Pandemic }\end{array}$} \\
\hline & $\begin{array}{l}\text { Crude B } \\
(95 \% \mathrm{CI})\end{array}$ & $\begin{array}{l}\text { Adjust B } \\
(95 \% \mathrm{CI})^{\mathrm{a}}\end{array}$ & $\begin{array}{l}\text { Crude B } \\
(95 \% \mathrm{CI})\end{array}$ & $\begin{array}{l}\text { Adjust B } \\
(95 \% \mathrm{CI})^{\mathrm{a}}\end{array}$ \\
\hline Baseline CAPE-PI5 total scores & $0.34(0.29,0.39)$ & $0.33(0.27,0.38)$ & $0.91(0.49,1.33)$ & $0.89(0.47,1.31)$ \\
\hline Baseline insomnia total scores & $0.00(-0.00,0.01)$ & $0.00(-0.01,0.01)$ & $0.43(0.36,0.49)$ & $0.42(0.36,0.49)$ \\
\hline Baseline CAPE-PI 5 scores $\times$ Baseline insomnia total scores & $0.00(-0.02,0.02)$ & $-0.00(-0.02,0.02)$ & $-0.04(-0.18,0.11)$ & $-0.03(-0.18,0.11)$ \\
\hline
\end{tabular}

Notes: ${ }^{a}$ Adjusting for demographic characteristics (sex, age, ethnicity, residence location, family income, parental marital status, "left-behind" child status, single child status, history of mental disorders, chronic physical illness, family history of mental disorders), and a two-sided $p<0.05$ was considered statistically significant.

Abbreviations: PLEs, psychotic-like experiences; CAPE-PI5, the 15-item positive subscale of the community assessment of psychic experiences. 
persistence of PLEs is affected by multiple factors. This can also be indirectly supported by the fact that all psychological interventions that greatly improve insomnia, have a limited effect on ameliorating PLEs. ${ }^{5}$

In this study, the reverse relationship was first explored between insomnia and PLEs. The presence of PLEs before the pandemic could predict the presence of insomnia during the pandemic, regardless of whether insomnia was experienced before the pandemic. One previous study has tested and verified the oppositional model in a small sample of the clinical population. ${ }^{35}$ Our research further extends the conclusion to the general population, suggesting that there is a mutual effect of insomnia and psychosis on a continuum where PLEs and clinical psychotic symptoms lie on the two ends.

This study also examined the correlation between symptom severity of insomnia and PLEs. Previous crosssectional research has suggested a dose-dependent relationship between insomnia chronicity and PLEs in the general population, ${ }^{36}$ with a similar effect size $(r=$ $0.33-0.41$ ) as found in our results. However, there is still a lack of longitudinal research on this predictive relationship. In this study, insomnia failed to predict the severity of PLEs, while PLEs and all three factors (PI, BEs and PAs) before the pandemic appeared to have moderate to strong correlations with the severity of insomnia during the pandemic. The results further suggest a higher complexity of mechanism for PLEs development. Insomnia may play an important role in the occurrence of PLEs. However, the impact of insomnia on the persistence and development of PLEs may be diluted by other internal and external environmental factors, ${ }^{37-39}$ which should be taken into account simultaneously in the intervention of PLEs. Additionally, the significant interaction between PAs and insomnia indicates that insomnia may aggravate hallucinations, rather than other types of PLEs.

There are several strengths in our study. The two waves of the survey before and during the COVID-19 pandemic provided an opportunity to explore the causal links between insomnia and PLEs. To our knowledge, this is the first study on the bidirectional temporal associations of insomnia and PLEs in the general population, as well as on their dose-dependent relationship in a longitudinal study, which will help elucidate the links between the mechanisms of insomnia and PLEs.

Some limitations also need to be noted for this study. First, there was a high attrition rate in the study, which may impact the accuracy of symptom prevalence. As the start of the 2020 spring semester was delayed due to the pandemic, we had to conduct the second wave of the survey through text messages or emails, resulting in a high attrition rate. Although no significant differences were found for the baseline prevalence between students who followed up and those lost to follow-up, the results need to be interpreted with caution. Meanwhile, due to the limited size of the final sample, we could not further explore the association between PLEs and insomnia in Hubei Province (a high-risk area during the COVID-19 pandemic). Third, PLEs were assessed using a selfreported web-based questionnaire, which may reduce the data's reliability. However, we adopted a cut-off value for PLEs that has been validated with interview-certified PLEs. We did not use a well-validated self-report measure to evaluate insomnia, and some important sleep-related variables (eg, sleep duration and impairment/distress of insomnia) were not considered. However, the three items have shown good applicability in the Chinese population in the previous research ${ }^{40}$ and acceptable internal consistency in this sample. The prevalence of insomnia before the pandemic was also comparable to previous data from the American Psychiatric Association. ${ }^{29}$ Finally, some other important potential confounders (eg, mood symptoms) were not considered in this study. However, as the mediating effects of negative affect have been certified in previous research, it may weaken the predictive effect of insomnia/PLEs on PLEs/insomnia to include these factors in the model.

In conclusion, there are bidirectional associations between insomnia and PLE for new-onset symptoms. However, while PLEs can also predict the persistence and severity of insomnia, the predictive effect of insomnia on PLEs is limited in the general population. Therefore, although insomnia is an important predictor for the occurrence of PLEs, a more complex mechanism may underlie the persistence and development of PLEs. Further validation is needed in large sample, as well as in mechanism research.

\section{Data Sharing Statement}

The data that support the findings of this study are available from the corresponding author upon reasonable request.

\section{Acknowledgments}

We wish to thank all the participants in this study. 


\section{Funding}

The study was supported by the Science and Technology Program of Guangzhou (No: 202102020702), the Guangzhou Municipal Psychiatric Disease Clinical Transformation Laboratory (No: 201805010009), Key Laboratory for Innovation platform Plan, Science and Technology Program of Guangzhou; Science and Technology Plan Project of Guangdong Province (No.2019B030316001); and Guangzhou municipal key discipline in medicine (2017-2019). The funding sources had no involvement in study design; the collection, analysis and interpretation of data; the writing of the report; or the decision to submit the article for publication.

\section{Disclosure}

The authors report no conflicts of interest in this work.

\section{References}

1. Barton J, Kyle SD, Varese F, Jones SH, Haddock G. Are sleep disturbances causally linked to the presence and severity of psychotic-like, dissociative and hypomanic experiences in non-clinical populations? A systematic review. Neurosci Biobehav Rev. 2018;89:119-131. doi:10.1016/j.neubiorev.2018.02.008

2. Reeve S, Sheaves B, Freeman D. The role of sleep dysfunction in the occurrence of delusions and hallucinations: a systematic review. Clin Psychol Rev. 2015;42:96-115. doi:10.1016/j.cpr.2015.09.001

3. Bordoloi M, Ramtekkar U. Relationship between sleep and psychosis in the pediatric population: a brief review. Med Sci (Basel). 2018;6 (3):76.

4. Laskemoen JF, Simonsen C, Büchmann C, et al. Sleep disturbances in schizophrenia spectrum and bipolar disorders - a transdiagnostic perspective. Compr Psychiatry. 2019;91:6-12. doi:10.1016/j. comppsych.2019.02.006

5. Waite F, Sheaves B, Isham L, Reeve S, Freeman D. Sleep and schizophrenia: from epiphenomenon to treatable causal target. Schizophr Res. 2020;221:44-56. doi:10.1016/j.schres.2019.11.014

6. González-Rodríguez A, Labad J, Seeman MV. Sleep disturbances in patients with persistent delusions: prevalence, clinical associations, and therapeutic strategies. Clocks Sleep. 2020;2(4):399-415. doi:10.3390/clockssleep2040030

7. Monti JM, Torterolo P, Perumal SRP. Sleep in schizophrenia patients and the effects of second generation antipsychotic drugs. In: Dopamine and Sleep. Springer International Publishing; 2016:213-233.

8. Yung AR, Nelson B, Baker K, Buckby JA, Baksheev G, Cosgrave EM. Psychotic-like experiences in a community sample of adolescents: implications for the continuum model of psychosis and prediction of schizophrenia. Aust N Z J Psychiatry. 2009;43 (2):118-128. doi:10.1080/00048670802607188

9. Linscott RJ, van Os J. An updated and conservative systematic review and meta-analysis of epidemiological evidence on psychotic experiences in children and adults: on the pathway from proneness to persistence to dimensional expression across mental disorders. Psychol Med. 2013;43(6):1133-1149. doi:10.1017/S0033291712001626

10. Sullivan SA, Kounali D, Cannon M, et al. A population-based cohort study examining the incidence and impact of psychotic experiences from childhood to adulthood, and prediction of psychotic disorder. Am J Psychiatry. 2020;177(4):308-317. doi:10.1176/appi.ajp.20 19.19060654
11. Healy C, Brannigan R, Dooley N, et al. Childhood and adolescent psychotic experiences and risk of mental disorder: a systematic review and meta-analysis. Psychol Med. 2019;49(10):1589-1599. doi:10.1017/S0033291719000485

12. Freeman D, Thompson C, Vorontsova N, et al. Paranoia and post-traumatic stress disorder in the months after a physical assault: a longitudinal study examining shared and differential predictors. Psychol Med. 2013;43(12):2673-2684. doi:10.1017/S0033291 $71300038 \mathrm{X}$

13. Freeman D, Stahl D, McManus S, et al. Insomnia, worry, anxiety and depression as predictors of the occurrence and persistence of paranoid thinking. Soc Psychiatry Psychiatr Epidemiol. 2012;47 (8):1195-1203. doi:10.1007/s00127-011-0433-1

14. Sheaves B, Bebbington PE, Goodwin GM, et al. Insomnia and hallucinations in the general population: findings from the 2000 and 2007 British Psychiatric Morbidity Surveys. Psychiatry Res. 2016;241:141-146. doi:10.1016/j.psychres.2016.03.055

15. Reeve S, Emsley R, Sheaves B, Freeman D. Disrupting sleep: the effects of sleep loss on psychotic experiences tested in an experimental study with mediation analysis. Schizophr Bull. 2018;44 (3):662-671.

16. WHO. Statement on the Second Meeting of the International Health Regulations (2005) Emergency Committee Regarding the Outbreak of Novel Coronavirus (2019-Ncov). WHO; 2020.

17. Zhou SJ, Zhang LG, Wang LL, et al. Prevalence and socio-demographic correlates of psychological health problems in Chinese adolescents during the outbreak of COVID-19. Eur Child Adolesc Psychiatry. 2020;29(6):749-758. doi:10.1007/s00787-02001541-4

18. Cao W, Fang Z, Hou G, et al. The psychological impact of the COVID-19 epidemic on college students in China. Psychiatry Res. 2020;287:112934. doi:10.1016/j.psychres.2020.112934

19. Torales J, O'Higgins M, Castaldelli-Maia JM, Ventriglio A. The outbreak of COVID-19 coronavirus and its impact on global mental health. Int J Soc Psychiatry. 2020;66(4):317-320. doi:10.1177/ 0020764020915212

20. Sun M, Wang D, Jing L, Zhou L. Changes in psychotic-like experiences and related influential factors in technical secondary school and college students during COVID-19. Schizophr Res. 2021;231:3-9. doi:10.1016/j.schres.2021.02.015

21. Sun M, Xue Z, Zhang W, et al. Psychotic-like experiences, trauma and related risk factors among "left-behind" children in China. Schizophr Res. 2017;181:43-48. doi:10.1016/j.schres.2016.09.030

22. Koyanagi A, Stubbs B, Lara E, et al. Psychotic experiences and subjective cognitive complaints among 224842 people in 48 lowand middle-income countries. Epidemiol Psychiatr Sci. 2018;29:e11. doi:10.1017/S2045796018000744

23. Koyanagi A, Stickley A. The association between sleep problems and psychotic symptoms in the general population: a global perspective. Sleep. 2015;38(12):1875-1885. doi:10.5665/sleep.5232

24. Capra C, Kavanagh DJ, Hides L, Scott J. Brief screening for psychosis-like experiences. Schizophr Res. 2013;149(1-3):104-107. doi:10.1016/j.schres.2013.05.020

25. Sun M, Wang D, Jing L, Xi C, Dai L, Zhou L. Psychometric properties of the 15-item positive subscale of the community assessment of psychic experiences. Schizophr Res. 2020;222:160-166. doi:10.1016/ j.schres.2020.06.003

26. Sun M, Wang D, Jing L, et al. Comparisons between self-reported and interview-verified psychotic-like experiences in adolescents. Early Interv Psychiatry. 2021. doi:10.1111/eip.13132

27. Corp IBM. IBM SPSS Statistics for Windows, Version 23.0. Armonk, NY, USA: IBM Corp; 2015.

28. Liu N, Zhang F, Wei C, et al. Prevalence and predictors of PTSS during COVID-19 outbreak in China hardest-hit areas: gender differences matter. Psychiatry Res. 2020;287:112921. doi:10.1016/j. psychres.2020.112921 
29. APA. Diagnostic and Statistical Manual of Mental Disorders, 5th Edition (DSM-5). Washington, DC: American Psychiatric Publishing; 2013.

30. Cénat JM, Blais-Rochette C, Kokou-Kpolou CK, et al. Prevalence of symptoms of depression, anxiety, insomnia, posttraumatic stress disorder, and psychological distress among populations affected by the COVID-19 pandemic: a systematic review and meta-analysis. Psychiatry Res. 2021;295:113599. doi:10.1016/j.psychres.2020.113599

31. Jahrami H, BaHammam AS, Bragazzi NL, Saif Z, Faris M, Vitiello MV. Sleep problems during the COVID-19 pandemic by population: a systematic review and meta-analysis. J Clin Sleep Med. 2021;17(2):299-313. doi:10.5664/jcsm.8930

32. Rossi R, Socci V, Talevi D, et al. COVID-19 pandemic and lockdown measures impact on mental health among the general population in Italy. Front Psychiatry. 2020;11:790. doi:10.3389/fpsyt.2020.00790

33. Huang Y, Zhao N. Generalized anxiety disorder, depressive symptoms and sleep quality during COVID-19 outbreak in China: a web-based cross-sectional survey. Psychiatry Res. 2020;288:112954. doi:10.1016/j.psychres.2020.112954

34. van Os J, Linscott RJ, Myin-Germeys I, Delespaul P, Krabbendam L. A systematic review and meta-analysis of the psychosis continuum: evidence for a psychosis proneness-persistence-impairment model of psychotic disorder. Psychol Med. 2009;39(2):179-195. doi:10.1017/ S0033291708003814

35. Reeve S, Nickless A, Sheaves B, Freeman D. Insomnia, negative affect, and psychotic experiences: modelling pathways over time in a clinical observational study. Psychiatry Res. 2018;269:673-680. doi:10.1016/j.psychres.2018.08.090
36. Freeman D, Brugha T, Meltzer H, Jenkins R, Stahl D, Bebbington P. Persecutory ideation and insomnia: findings from the second British National Survey of Psychiatric Morbidity. J Psychiatr Res. 2010;44 (15):1021-1026. doi:10.1016/j.jpsychires.2010.03.018

37. Madrid-Gambin F, Focking M, Sabherwal S, et al. Integrated lipidomics and proteomics point to early blood-based changes in childhood preceding later development of psychotic experiences: evidence from the Avon longitudinal study of parents and children. Biol Psychiatry. 2019;86:25-34. doi:10.1016/j.biopsych.2019.01.018

38. Kalman JL, Bresnahan M, Schulze TG, Susser E. Predictors of persisting psychotic like experiences in children and adolescents: a scoping review. Schizophr Res. 2019;209:32-39. doi:10.1016/j. schres.2019.05.012

39. Solmi F, Colman I, Weeks M, Lewis G, Kirkbride JB. Trajectories of neighborhood cohesion in childhood, and psychotic and depressive symptoms at age 13 and 18 years. J Am Acad Child Adolesc Psychiatry. 2017;56(7):570-577. doi:10.1016/j.jaac.2017.04.003

40. Wang D, Ross B, Zhou X, et al. Sleep disturbance predicts suicidal ideation during COVID-19 pandemic: a two-wave longitudinal survey. $J$ Psychiatr Res. 2021;143:350-356. doi:10.1016/j. jpsychires.2021.09.038

\section{Publish your work in this journal}

Nature and Science of Sleep is an international, peer-reviewed, open access journal covering all aspects of sleep science and sleep medicine, including the neurophysiology and functions of sleep, the genetics of sleep, sleep and society, biological rhythms, dreaming, sleep disorders and therapy, and strategies to optimize healthy sleep.
The manuscript management system is completely online and includes a very quick and fair peer-review system, which is all easy to use. Visit http://www.dovepress.com/testimonials.php to read real quotes from published authors. 